\title{
Novel global sensitivity analysis methodology accounting for the crucial role of the distribution of input parameters: application to systems biology models
}

\author{
Maria Rodriguez-Fernandez ${ }^{12 *}$, Julio R. Banga ${ }^{3}$ and Francis J. Doyle III ${ }^{12}$ \\ ${ }^{1}$ Department of Chemical Engineering, University of California Santa Barbara, CA 93106-5080, USA \\ ${ }^{2}$ Institute for Collaborative Biotechnologies, University of California Santa Barbara, CA 93106-5100, USA \\ ${ }^{3}$ (Bio)Process Engineering Group, IIM-CSIC, C/Eduardo Cabello 6, 36208 Vigo, Spain
}

\begin{abstract}
SUMMARY
The reliability of model predictions is affected by multiple sources of uncertainty, therefore most of the efforts for modeling biological systems include a sensitivity analysis step aiming to identify the key contributors to uncertainty. This generates insight about the robustness of the model to variations in environmental conditions, kinetic parameters, initial concentration of the species or any other source of uncertainty. Local sensitivities measure the robustness of the model to small perturbations on the inputs around their nominal value. There are several numerical methods for the calculation of local sensitivities, but the calculated values should be identical within the numerical accuracy of the method used. In contrast, as will be shown in this contribution, the results of different global sensitivity analysis (GSA) methods can be very different and highly dependent on the distribution considered for the inputs under evaluation. In this work, derivative based global sensitivities are extended to be able to consider an accurate probability density function for the parameters based on the likelihood function. This strategy enforces the areas of the parameter space most likely to reproduce the desired behavior minimizing the importance of parameter sets with low probability of being optimal to dominate the sensitivity ranking. A model of a biochemical pathway with three enzymatic steps is used here to illustrate the performance of several relevant GSA methods considering different probability density functions for the parameters and revealing important hints about which method and distribution to choose for each type of model and purpose of the analysis.

Copyright (C) 2011 John Wiley \& Sons, Ltd.
\end{abstract}

Received ...

KEY WORDS: Global sensitivity analysis; parameter distribution; model identification; parameter estimation; robustness; systems biology

\section{INTRODUCTION}

Pareto's law (also known as the law of the "vital few and trivial many") states that, for many events, roughly $80 \%$ of the effects come from $20 \%$ of the causes. This behavior has also been recorded in the field of modeling where the input factors' importance has been found to be unevenly distributed, with a few factors creating almost all the uncertainty and the majority making only a negligible contribution [1]. This property motivates the determination of the most relevant inputs that can ultimately lead to model simplification if negligible factors are found.

Sensitivity analysis (SA) and uncertainty analysis are related methods that ideally should be run in tandem. While uncertainty analysis focuses on quantifying uncertainty in the model output, SA

\footnotetext{
${ }^{*}$ Correspondence to: Department of Chemical Engineering, University of California Santa Barbara, CA 93106-5080,

USA. E-mail: mrodriguez@engineering.ucsb.edu 
could be defined as "the study of how uncertainty in the output of a model can be apportioned to different sources of uncertainty in the model input" [2].

In most of the available systems biology literature, sensitivities are understood as derivatives of a particular state with respect to a particular input, and the influence of factors on states is assessed by looking at a single point in the input space. However, global sensitivity analysis (GSA) methods that investigate the sensitivity over the entire input space have been recently studied with application to biochemical models [3] and several tools facilitating the computation of the most known sensitivity measures have been developed, namely Systems Biology Toolbox 2 [4], COPASI [5], SBML-SAT [6], PottersWheel [7] and SensSB [8]. Nevertheless, a SA method can fail if its underlying purpose is vaguely defined; diverse tests and measures may be thrown at a problem, producing a range of different input rankings but leaving the researcher none the wiser as to which ranking to believe or favor [1]. Several studies on biological systems have shown that the ranking of the parameter sensitivity may largely depend on the analysis method $[9,10,11]$ but the reasons for these discrepancies have not been widely examined. Moreover, the distribution of input factors has a large impact on the result of any GSA method and in the case of biological systems these distributions are not always easy to assess.

In this work, we review several local and global sensitivity analysis techniques (Morris method, Sobol' indices and derivative based global sensitivities) and we study their adequacy for different applications. The crucial influence of the input factors distribution is analyzed and illustrated with a benchmark case study regarding a biochemical pathway model. Moreover, we introduce a novel GSA methodology that is able to account for the crucial role of the distribution of input parameters when experimental data are available.

\section{METHODOLOGY}

In order to be able to make use of the different sensitivity analysis techniques as defined above, we first have to identify the type of models under investigation and the input and output factors of interest.

\subsection{Mathematical models}

Many dynamic models of biological processes together with the input-output experiment designed for their identification can be described by a general nonlinear model. Let $\boldsymbol{x}$ be the vector of $N_{x}$ state variables and $\boldsymbol{p}$ the vector of $N_{p}$ model parameters:

$$
\begin{aligned}
\dot{\boldsymbol{x}}(\boldsymbol{p}, t) & =f[\boldsymbol{x}(\boldsymbol{p}, t), \boldsymbol{u}(t), \boldsymbol{p}], \boldsymbol{x}(0)=\boldsymbol{x}_{0} \\
\boldsymbol{y}(\boldsymbol{p}, t) & =g[\boldsymbol{x}(\boldsymbol{p}, t), \boldsymbol{u}(\boldsymbol{p}, t), \boldsymbol{p}]
\end{aligned}
$$

Note that $f$ specifies the model, $\boldsymbol{u}$ specifies the vector of $N_{u}$ control variables for a particular experiment and $\boldsymbol{y}$ the vector of $N_{y}$ measured states (output variables). An experiment is specified by the initial conditions $\boldsymbol{x}(0)$, the control variables $\boldsymbol{u}$ and the observations $\boldsymbol{y}$.

\subsection{Input and output variables}

Once the model has been defined, the next elements to be identified prior to sensitivity analysis are the input and output variables of interest. In mathematical models of biological systems the output variables are usually the concentration (or a function of them) of the measurable biological components (proteins, metabolites, transcriptions factors, etc.). Depending on the target of the analysis, a certain behavior of a set of states is under investigation, such as the agreement with available experimental data or certain qualitative dynamics (steady states, monotonicity, oscillations, etc.).

Given a nonlinear dynamic model representing several components, the set of states under analysis and the time points where the sensitivities are evaluated will affect the results of the importance ranking. Often, the sensitivity of each output factor with respect to each input factor is evaluated at the given sampling points but when the problem size increases, this information 
has to be summarized for the sake of clarity. The way of computing these metrics (averaging the sensitivities over time or focusing on other regions as maximum values or values at the steady state; using relative or absolute values with respect to the output and/or input factors, etc.) will strongly influence the results so particular attention should be payed.

Most of the sensitivity analyses performed on biological systems are focused on the model parameters $\boldsymbol{p}$. In the case of an ordinary differential equation (ODE) model derived by the principle of mass action from a network of biochemical reactions, the parameters are typically rate constants (e.g. association rates, disassociation rates, catalytic rates) but models are not always deduced from mass action, and other parameters such as Hill coefficients or Michaelis-Menten constants can be found. Another common source of uncertainty are the initial values of the state variables $\boldsymbol{x}(0)$. Other variables affecting the model, including environmental or experimental conditions, can be treated as control variables $\boldsymbol{u}$.

\subsection{Input factors distribution}

One of the most important stages in GSA is to determine the distribution of input factors [12]. Making reasonable assumptions about the a priori distribution of the initial values of the state variables or the uncertainty in the control variables is often feasible since most of the time they can be measured directly. On the contrary, evaluating the distribution of the model parameters that can rarely be determined experimentally is much more challenging. Ideally, the distribution of the parameters can be inferred by estimation from the available experimental data and it can be stated that the parameters present a certain distribution with a mean $\bar{p}$ and standard deviation $\sigma$. However, this is not always possible either because we want to perform the sensitivity analysis prior the parameter estimation, the distribution cannot be easily characterized with the available data, or we want to analyze the sensitivity of the model in broader parameter spaces. In these cases, a common practice is to select wide bounds and assume that the parameters are uniformly distributed among these values. The selection of the lower bounds is usually easy because of the physical or biological meaning (e.g. a kinetic constant cannot be negative). However, the upper bounds are often chosen arbitrarily to contain all the potential values for each parameter and, therefore, it is expected that good solutions may lie closer to the lower bounds than to the upper bounds [13]. In this situation, a uniform distribution will not intensively explore the regions with small values whereas a logarithmic distribution will do. Moreover, a logarithmic distribution is also helpful in the case of variables that can intrinsically have values in different orders of magnitude (for instance, pre-exponential factors in kinetic equations or degradation constants of different species) or with variables without physical or biological meaning, for which selecting bounds is a difficult task. Figure 1 illustrates this situation for a variable spanning from $10^{-3}$ to $10^{3}$. If we generate a number of sampling points between

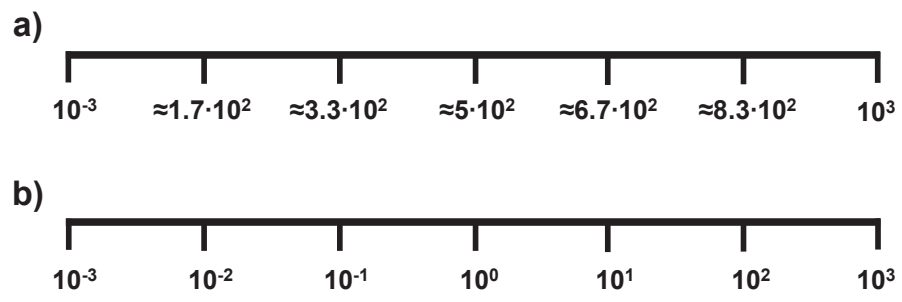

Figure 1. Intervals within a variable range:

a) Uniform distribution. b) Logarithmic distribution.

these bounds using a uniform distribution, we will approximately obtain the same number of points in every interval depicted in Figure 1a. Alternatively, if a logarithmic distribution is selected, the points will have equal probability across the sub-ranges shown in Figure 1b. In other words, with a uniform distribution, roughly $90 \%$ of the sampling points will have values between $10^{2}$ and $10^{3}$ with approximately only $0.1 \%$ of the sampling points covering the area between $10^{-3}$ and 1 . 


\subsection{Review of available sensitivity analysis methods}

Local sensitivity indices are computed at the nominal values used for the parameters and the behavior of the response function is described only locally in the input space. Moreover, preliminary experiments and model calibration tests should be carried out in order to obtain a first guess for the parameter values and an iterative scheme involving both steps is required in order to study the model sensitivity. In addition, these methods are linear thus they are not sufficient for dealing with complex models, especially those in which there are nonlinear interactions between parameters.

In contrast, global sensitivity analysis methods evaluate the effect of a parameter while all other parameters are varied simultaneously, accounting for interactions between parameters without depending on the stipulation of a nominal point (they explore the entire range of each parameter). Among the most widely used GSA methods are the ones studied in the following: the Morris method [14] mostly used as a screening method, the Sobol' method [15] considered as one of the most powerful despite its high computational cost and the derivative based global sensitivity measures (DGSM) [16] based on averaging the local sensitivities over the parameter space.

2.4.1. Local sensitivity methods. Most of the sensitivity analysis described in the literature are based on derivatives. Indeed the partial derivative $\partial y_{i} / \partial p_{j}$ of an output $y_{i}$ with respect to an input $p_{j}$ can be thought of as a mathematical definition of the sensitivity of $y_{i}$ versus $p_{j}$ (for the sake of simplicity, in the rest of this paper we will denote all the input variables by $p$ no matter whether they are parameters, initial values or control variables).

Local sensitivity coefficients are the partial derivatives of the model state variables to the model inputs evaluated at the normal operating point where all the input variables have their nominal values, $\hat{\boldsymbol{p}}$. Considering the model described by Eq. 1-2, the sensitivity coefficients that form the sensitivity matrix are:

$$
S_{a b s, i j}(\boldsymbol{p})=\left(\frac{\partial y_{i}}{\partial p_{j}}\right)_{\boldsymbol{y}=\boldsymbol{y}(t, \hat{\boldsymbol{p}}), \boldsymbol{p}=\hat{\boldsymbol{p}}}
$$

These sensitivities are denoted absolute sensitivity functions and are useful for calculating errors due to input factors variations and for assessing the times at which an input has its greatest or least effect. However, absolute sensitivity functions are not normalized and they are not useful for comparing the effects of different input factors for what relative sensitivity functions, computed by multiplying the partial derivative (the absolute sensitivity function) by the nominal value of the input and dividing by the output value, should be used. The relative sensitivity of the function $y_{i}$ to variations in the parameter $p_{j}$ is given by:

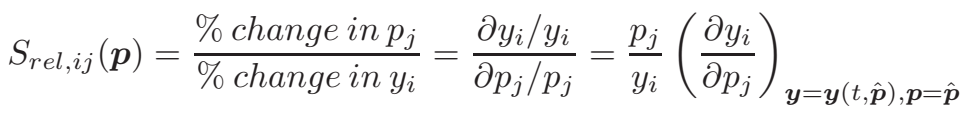

Relative sensitivities are ideal for comparing parameters, because they are dimensionless, normalized functions. However, they present division by zero problems when $y_{i}$ is equal to zero and gives undue weight to the response if it is small. That is why, a threshold value $\left(\boldsymbol{y}_{\min }\right)$ should be defined to be used in the premultiplicator factor in Eq. (4) when the latter is smaller than the $\boldsymbol{y}_{\text {min }}$ value [17].

There are several numerical methods for the calculation of local sensitivities (e.g. finite differences [18], iterative approximation with directional derivatives [19], direct decoupled method [20]) but the calculated values should be identical within the numerical accuracy of the method used.

2.4.2. Morris method. The Morris method is traditionally used as a screening sensitivity technique for problems with large number of variables (100s, 1000s) and/or for CPU intensive models. It is composed of individually randomized "one-factor-at-a-time" experiments. Each input factor is assumed to be uniformly distributed in the interval $[0,1]$ and may take a discrete number of values, called levels. The range of each input is divided into $k$ levels then the region of experimentation $\Omega$ is a $N_{p}$-dimensional k-level grid. 
The sensitivity measures proposed in the original work of Morris [14] are based on what is called an elementary effect (EE). The EE of the $j$-th input factor on the $i$-th input factor is defined as:

$$
E E_{i j}(\hat{\boldsymbol{p}})=\frac{\left[y_{i}\left(\hat{p}_{1}, \ldots, \hat{p}_{j-1}, \hat{p}_{j}+\Delta, \hat{p}_{j+1}, \ldots, \hat{p}_{N_{p}}\right)-y_{i}(\hat{\boldsymbol{p}})\right]}{\Delta}
$$

where $\Delta$ is a predetermined multiple of $1 /(k-1)$ and point $\hat{\boldsymbol{p}} \in \Omega$ is such that $\hat{\boldsymbol{p}}+\Delta$ is still in $\Omega$.

The distribution of elementary effects $F_{i j}$ is obtained by randomly sampling $N$ points from $\Omega$. Two sensitivity measures are evaluated for each factor: $\mu_{i j}$ an estimate of the mean of the distribution $F_{i j}$, and $\sigma_{i j}$ an estimate of the standard deviation of $F_{i j}$. A high value of $\mu_{i j}$ indicates a parameter with an important overall influence on the output. A high value of $\sigma_{i j}$ indicates a parameter involved in interactions with other parameters or whose effect is nonlinear. Non-monotonic functions have regions of positive and negative values of partial derivatives $E E_{i j}(\hat{\boldsymbol{p}})$, hence due to the effect of averaging, $\mu_{i j}$ can be very small or even zero. To avoid such situations Campolongo et al. [21] considered another sensitivity measure, $\bar{\mu}_{i j}$, which is an estimate of the mean of the distribution of the absolute values of the elementary effects and showed that $\bar{\mu}_{i j}$ gives a better estimate of the order of importance than $\mu_{i j}$.

The total computational cost for the simplest form of this method for each distribution $F_{i j}$ is $N_{F}=2 N N_{p}$ (each elementary effect requires the computation of $y_{i}$ twice). Morris [14] suggested a more economical algorithm by using already computed values of functions in the calculation of more than one elementary effect that has a cost of $N_{F}=N\left(N_{p}+1\right)$. Previous experiments [1] have demonstrated that the choice $k=4$ and $N=10$ produces valuable results.

Due to the frequent dispersity of biochemical parameters argumented before, in this work the factors are assumed to be uniformly distributed in $[0,1]$ and then transformed from the unit hypercube to a logarithmic distribution. This strategy aims to explore the parameter space in a more effective way when it spans over several orders of magnitude.

2.4.3. Variance based methods. The most widely used variance based methods are the extended FAST [22] and the Sobol' method [15] considered as one of the most powerful despite its high computational cost. The basis of the Sobol' method is the decomposition of the variance of the model output function (D) into summands of variances of combinations of input parameters in increasing dimensionality. The variances of the terms in the ANOVA decomposition add up to the total variance of the function:

$$
D=\sum_{s=1}^{N_{p}} \sum_{j_{1}<\ldots<j_{s}}^{N_{p}} D_{j_{1} \ldots j_{s}}
$$

where $D_{j_{1} \ldots j_{s}}$ are called partial variances. Sobol' defined the global sensitivity indices as the ratios:

$$
S I_{j_{1} \ldots j_{s}}=D_{j_{1} \ldots j_{s}} / D
$$

Therefore, all $S I_{j_{1} \ldots j_{s}}$ are nonnegative and add up to one. There are $2^{N_{p}}$ terms in this summation and the calculation of all of them is sometimes not practical. For this reason Sobol' introduced sensitivity indices for subsets of variables and the total sensitivity indices accounting for the interaction between parameters defined as:

$$
S I_{j}^{T}=S I_{j}+\sum_{s=1}^{N_{p}} S I_{j s}+\ldots+S I_{1,2, \ldots, N_{p}}
$$

The first-order sensitivity indices $\left(S I_{j}\right)$ account only for the effect of the parameter $p_{j}$ and the total sensitivity indices $\left(S I_{j}^{T}\right)$ account for the effect that $p_{j}$ and its interactions with the rest of the parameters have on the model output. Sobol' [15] found an elegant way of computing these indices 
directly from the model $f(\boldsymbol{p})$. This method was improved later by Saltelli [23] leading to:

$$
\begin{aligned}
S I_{j} & =\frac{\int_{H^{N_{p}}}\left[f(\boldsymbol{p}) f\left(p_{j}, \boldsymbol{p}_{-j}^{\prime}\right)-f\left(\boldsymbol{p}^{\prime}\right)\right] d \boldsymbol{p} d \boldsymbol{p}^{\prime}}{\int_{H^{N_{p}}} f^{2}(\boldsymbol{p}) d \boldsymbol{p}-f_{0}^{2}(\boldsymbol{p})} \\
S I_{j}^{T} & =\frac{1}{2} \frac{\int_{H^{N_{p}}}\left[f\left(\boldsymbol{p}^{\prime}\right)-f\left(p_{j}, \boldsymbol{p}_{-j}^{\prime}\right)\right]^{2} d \boldsymbol{p} d \boldsymbol{p}^{\prime}}{\int_{H^{N_{p}}} f^{2}(\boldsymbol{p}) d \boldsymbol{p}-f_{0}^{2}(\boldsymbol{p})}
\end{aligned}
$$

where $\boldsymbol{p}$ and $\boldsymbol{p}^{\prime}$ are two independent random points in $H^{N_{p}}$ and $\left(p_{j}, \boldsymbol{p}_{-j}^{\prime}\right)$ is the result of taking the element $p_{j}$ from $\boldsymbol{p}$ and replacing it into $\boldsymbol{p}^{\prime}$. However, variance based methods generally require a large number of function evaluations to achieve reasonable convergence and can become impractical for large systems biology problems.

2.4.4. Global derivative based methods. As already mentioned, local sensitivity indices are questionable when the model input is uncertain and when the model is of unknown linearity. To circumvent this limitation, different authors suggested to use the average of the local sensitivities over the parameter space. This approach was formalized by Kucherenko et al. [16] who called the resulting set of measures derivative based global sensitivity measures (DGSM), using an analogy with variance based global sensitivity measures. A link between DGSM and Sobol' indices was established in [24].

DGSM are based on averaging the local sensitivity measures $S_{i j}(\boldsymbol{p})$ over the parameter space $H^{N_{p}}$. As explained for the Morris method, non-monotonic functions may have positive and negative partial derivatives, thus, averaging the values can lead to misleading results so measures based on the absolute value of $\left|S_{i j}(\boldsymbol{p})\right|$ should be used. Such measures can be defined as:

$$
M_{i j}=\int_{H^{N_{p}}}\left|S_{i j}(\boldsymbol{p})\right| d p
$$

Nonlinear and interaction effects can be captured by the variance of $M_{i j}$ :

$$
\Sigma_{i j}=\sqrt{\int_{H^{N_{p}}}\left(\left|S_{i j}(\boldsymbol{p})\right|-M_{i j}\right)^{2} d p}
$$

$\Sigma_{i j}$ can also be presented as:

$$
\Sigma_{i j}^{2}=\int_{H^{N_{p}}}\left|S_{i j}(\boldsymbol{p})\right|^{2} d p-M_{i j}^{2}
$$

Note that in this technique the individual effects are evaluated as strict local derivatives with small increments unlike the incremental ratios of the Morris method.

\subsection{DGSM in a Bayesian framework}

In addition to the increasing use of well established global sensitivity analysis, several novel methodologies have been developed for assessing input sensitivities in systems biology models. Cho et al. [25] evaluated the global sensitivities by comparing the distributions of the parameters associated to acceptable and unacceptable sets with respect to a threshold objective function value. By checking the correlation coefficients of the corresponding cumulative frequencies each parameter is classified as insensitive if the two distributions are not similar or sensitive otherwise. Bentele et al. [26] calculated a measure of the global sensitivity from a weighted average of the absolute value of the relative local sensitivities over the parameter space (the weighting factor aims to amplify the statistical impact of sensitivities for those parameter sets that are more consistent with the experimental observations and therefore more probable). Zheng et al. [9] proposed a GSA method embedded within a random-search-based parameter identification routine (PID-embedded method) that calculates the sensitivity indices directly from the parameter sets generated by a Genetic Algorithm during parameter optimization. 
All these methods make use of the information available in form of experimental data to focus the sensitivity analysis on regions of the parameter space that are more plausible to fit the data. In this work, we suggest a method that enhances the benefits of exploiting the available information by computing the prior distribution of the parameters and integrating the derivative based sensitivity measures (DGSM) taking it into account. The derived metrics, $M_{B, i j}$ and $\Sigma_{B, i j}^{2}$, can be considered as probabilistic or Bayesian sensitivity analysis indices so we will call them Bayesian DGSM.

Sensitivity analysis is often related to parameter estimation through the identification of crucial parameters and the reduction of the model by fixing nonessential ones. Several estimator functions have been suggested as metrics for parameter estimation in the field of systems biology, most notably the maximum likelihood estimator introduced by Fisher in 1912 [27], for being the one that maximizes the probability of the observed event. The use of this estimator requires several assumptions including no modeling errors and Gaussian or known variance for the measurements (see [28] for details). Moreover, the maximum likelihood function, $J$ represents the probability density function (PDF) of a model for the occurrence of the measurements for given parameters. This function depends on the probability of the measurements and is given as:

$$
J(\boldsymbol{p})=\prod_{i=1}^{N E} \prod_{j=1}^{N V_{i}} \prod_{k=1}^{N M_{i j}} \frac{1}{\sqrt{\left(2 \pi \sigma_{i j k}^{2}\right)}} \exp \left[-\frac{\left(\tilde{y}_{i j k}-y_{i j k}(\boldsymbol{p})\right)^{2}}{2 \sigma_{i j k}^{2}}\right]
$$

where $\boldsymbol{p}$ is set of parameters under analysis; $i, j, k$ are indices for the experiments, variables, and time points, respectively; $N E$ is the number of experiments; $N V_{i}$ is the number of measured variables in experiment $i ; N M_{i j}$ is the number of measures of the variable $j$ during experiment $i ; \sigma_{i j k}^{2}$ is the variance of the measure $k$ of the variable $j$ in experiment $i$; $\tilde{y}_{i j k}$ is the measure $k$ of the variable $j$ in experiment $i ; y_{i j k}$ is the model predicted value $k$ of the variable $j$ in experiment $i$.

In this work we suggest the computation of the maximum likelihood function for a discrete number $(N)$ of parameter sets, therefore, the PDF is a representative of the probability of the available measurements to be generated by a particular set. In order to generate a discrete probability distribution:

$$
\sum_{q=1}^{N} p\left(\boldsymbol{p}_{q}\right)=1
$$

where $p_{q}$ is the $q^{t h}$ realization of $\boldsymbol{p}$ defined as a discrete-value random variable. Thus the probability of $\boldsymbol{p}_{q}$ can be defined as:

$$
p\left(\boldsymbol{p}_{q}\right)=\frac{J\left(\boldsymbol{p}_{q}\right)}{\sum_{q=1}^{N} J\left(\boldsymbol{p}_{q}\right)}
$$

and it can be used in the evaluation of the Bayesian DGSM as detailed in the following section.

\subsection{Evaluation of the integrals}

The computation of Bayesian DGSM, as well as Sobol' indices and regular DGSM is based on the evaluation of a series of integrals that can be presented in the following generic form:

$$
I[f]=\int_{H^{N_{p}}} f(\boldsymbol{p}) d p
$$

It is assumed that the function $f(\boldsymbol{p})$ is integrable in the $N_{p}$-dimensional unit hypercube $H^{N_{p}}$.

Classical grid methods for evaluating integrals become inefficient in high-dimensions because of the "curse of dimensionality" (exponential grows of the required integrand evaluations) [29]. Monte Carlo (MC) methods do not depend on the dimensionality and are effective in high dimensional integrations. However, the efficiency of MC methods is determined by the properties of random numbers that are known to be prone to clustering. A higher rate of convergence can be obtained by using deterministic uniformly distributed sequences also known as low-discrepancy sequences (LDS) instead of random numbers. Methods based on the usage of such sequences are known 
as Quasi Monte Carlo (QMC) methods. LDS are specifically designed to place sample points as uniformly as possible. Unlike random numbers, successive LDS points "know" about the position of previously sampled points and "fill" the gaps between them. There are a few well-known and commonly used LDS, also known as quasi random numbers, and different principles were used for their construction by Holton, Faure, Sobol', Niederreiter and others. Many practical studies have proven that Sobol' LDS are in many aspects superior to other LDS [29].

For a random variable that is uniformly distributed in $H^{N_{p}}$

$$
I[f]=E[f(\boldsymbol{p})]
$$

where $E[f(\boldsymbol{p})]$ is the mathematical expectation. An approximation of this expectation is

$$
I_{N}[f]=\sum_{q=1}^{N} f\left(\boldsymbol{p}_{q}\right) p\left(\boldsymbol{p}_{q}\right)
$$

where $\boldsymbol{p}_{q}$ is a sequence of LDS points of length $N$ uniformly distributed in a unit hypercube $H^{N_{p}}$.

Assuming equiprobable sampling points, $p\left(\boldsymbol{p}_{q}\right)=1 / N$. Therefore, from Eq. (9-10) and applying the QMC algorithm for the evaluation of the integrals to each output variable $y_{i}$ instead of $f$, the Sobol' indices can be calculated in a straightforward manner according to the formulae:

$$
\begin{aligned}
S I_{i j} & =\frac{\frac{1}{N} \sum\left[y_{i}(\boldsymbol{p}) y_{i}\left(p_{j}, \boldsymbol{p}_{-j}^{\prime}\right)-y_{i}\left(\boldsymbol{p}^{\prime}\right)\right]}{\frac{1}{N} \sum y_{i}^{2}(\boldsymbol{p})-\left(\frac{1}{N} \sum y_{i}(\boldsymbol{p})\right)^{2}} \\
S I_{i j}^{T} & =\frac{1}{2} \frac{\frac{1}{N} \sum\left[y_{i}\left(\boldsymbol{p}^{\prime}\right)-y_{i}\left(p_{j}, \boldsymbol{p}_{-j}^{\prime}\right)\right]^{2}}{\frac{1}{N} \sum y_{i}^{2}(\boldsymbol{p})-\left(\frac{1}{N} \sum y_{i}(\boldsymbol{p})\right)^{2}}
\end{aligned}
$$

Thus, each Quasi Monte Carlo sample point requires three computations of the model $y_{i}(\boldsymbol{p}), y_{i}\left(\boldsymbol{p}^{\prime}\right)$ and $y_{i}\left(p_{j}, \boldsymbol{p}_{-j}^{\prime}\right)$. For the computation of the Sobol' indices of an entire set of $N_{p}$ parameters, using $N$ sample points, the number of function evaluations is $N_{F}=N\left(N_{p}+2\right)$.

In the same way, applying the QMC algorithm to Eq. (11-13) and assuming that all the sampling points have equal probability, the DGSM can be easily computed as:

$$
\begin{aligned}
M_{i j} & =\frac{1}{N} \sum_{q=1}^{N}\left|S_{i j}\left(\boldsymbol{p}_{q}\right)\right| \\
\Sigma_{i j}^{2} & =\frac{1}{N} \sum_{q=1}^{N}\left|S_{i j}\left(\boldsymbol{p}_{q}\right)\right|^{2}-M_{i j}^{2}
\end{aligned}
$$

The computation of the DGSM using $N$ sample points requires the evaluation of the local sensitivities $N$ times.

Bayesian DGSM takes into account the prior distribution of the parameters, therefore $M_{B, i j}$ and $\Sigma_{B, i j}$ can be computed as:

$$
\begin{aligned}
M_{B, i j} & =\sum_{q=1}^{N}\left|S_{i j}\left(\boldsymbol{p}_{q}\right)\right| p\left(\boldsymbol{p}_{q}\right) \\
\Sigma_{B, i j}^{2} & =\sum_{q=1}^{N}\left|S_{i j}\left(\boldsymbol{p}_{q}\right)\right|^{2} p\left(\boldsymbol{p}_{q}\right)-M_{B, i j}^{2}
\end{aligned}
$$

\subsection{Extension to complex models}

In the case of complex models with multiple inputs and multiple outputs, large sensitivity matrices can be obtained and summary indices need to be computed to better interpret the results. When dealing with systems of ordinary differential equations (ODEs) or differential algebraic equations 
(DAEs), sensitivity indices of every observed state variable at each time point measurement with respect to each of the parameters are usually evaluated. In order to summarize all this information, sensitivity indices as the average of all the $S_{i j}$ for each parameter can be defined:

$$
S_{j}=\frac{1}{N_{x}} \frac{1}{N_{t}} \sum_{i=1}^{N_{x}} \sum_{k=1}^{N_{t}} S_{i j}\left(t_{k}\right)
$$

where $N_{x}$ is the number of measured states and $N_{t}$ the number of time points.

The same expression is applicable to $\bar{\mu}_{i j}, \sigma_{i j}, S I_{i j}, S I_{i j}^{T}, D_{i j}, M_{i j}, \Sigma_{i j}, M_{B, i j}$ and $\Sigma_{B, i j}$. However, different indices have particular features and they handle differently the effects of averaging information as discussed below.

Sobol' indices, are based upon the fraction of related partial variances in the overall variance, that is, they provide information about the sensitivity (how the uncertainty is distributed among the parameters) but not the magnitude of the uncertainty that is assessed by the total variance $D_{i}$. Thus, this summary will provide an average of the contribution to the total variance for each output variable at each sampling point. When an overall insignificant parameter is the most important in unsensitive regions of the time space or ranks very high with respect to an output variable that shows low uncertainty, the results can be misleading. Furthermore, when the value of the total variance $D_{i}$ has large variability among the different states or sampling points this ranking can be difficult to interpret. On the other hand, this normalization is sometimes useful since large discrepancies on the total variance of different states or even the same state in different time points can be due to state variables ranging over different orders of magnitude and the percentage of change can be of interest. Therefore, we recommend the computation of the total variance $D_{i}$ together with the indices $S I_{i j}$ and $S I_{i j}^{T}$ to get a deeper insight into the input factors importance.

Local sensitivities, Morris metrics, DGSM and Bayesian DGSM combine uncertainty with sensitivity analysis. $S_{i j}, \mu_{i j}, M_{i j}$ and $M_{B, i j}$ provide information about the relative importance of each parameter, but since they are not normalized metrics, the sum of them over $j$ (i.e. for the $N_{p}$ parameters) are informative measures of the uncertainty of a particular output in a certain time point. However, when the order of magnitude of the measured state variables is significantly different, the resulting averaged measures can be misleading. Depending on the purpose of the analysis, the use of relative values of the sensitivities may be advisable for computing these indices. If the sensitivity analysis is performed prior parameter estimation, similar measures should be used. That is, if a weighted least squares function will be used for the estimation, the relative sensitivities would be a better proxi of the parameter ranking. In contrast, if the optimization will be based on a standard least squares metric, the use of absolute sensitivities is advisable.

The use of relative sensitivities with respect to the input and output variance, could in principle overcome the main drawback of standard DGSM that provide the same measures for indices with different uncertainty when they have the same structural role in the model equations. However, we have found that the use of this normalization when some of the output states have small uncertainty can lead to wrong conclusions overweighting the importance of the parameters affecting these states even if the overall importance is small.

Biological networks often involve a wide range of time scales throughout the network. To avoid the side effects of averaging information of events occurring over different time scales, the experimental sampling points should be used as the times when the sensitivity is computed; therefore different time points will be required for states that change at different time scales. Assuming that the experimentalists have set up an appropriate experiment taking into consideration the different time scales (e.g. an enzyme binding event will be measured at shorter intervals than protein synthesis), the resulting sensitivity indices will accurately average the information from each of the species involved in the experiment.

\subsection{Applications of sensitivity analysis in systems biology}

Local sensitivity analysis have been widely applied in the field of systems biology to identify critical factors controlling biological behavior, design and optimize genetic circuits, analyze the tradeoff 
between robustness and fragility in cell regulation, and find appropriate drug targets [30]. However, a small but rapidly increasing number of studies in this area made use of global sensitivity measures $[25,26,31,32,33,34,11,35]$.

The main application of GSA consists on assessing the effect of parameter uncertainty in the model outcome that can be useful for establishing a ranking of importance of the parameters [11], identifying the critical parameters for a subsequent parameter estimation, model reduction by fixing unessential parameters, suggesting new experimental designs by determining the critical variables that capture the essential characteristics of the system [25, 32], etc.

Other applications can be found in [35] where a method based on GSA was used to determine the pseudo-global a priori identifiability of a model describing the aggregation of tau protein. A weighted average of a large number of correlation matrices calculated from the local sensitivities at quasi-random points in the relevant parameter space was used to remove the parameters that appeared to be highly correlated.

In addition, several attempts to overcome the drawbacks of optimal experimental design (OED) based on the Fisher Information Matrix (FIM) due to its local nature have been suggested [32, 34, 8]. In these approaches, the input factors for the GSA are no longer the parameters of the model but the experimental conditions such as initial values for the state variables, control variables profiles, sampling scheme, etc.

Other promising applications of global sensitivity analysis in the field of systems biology are: robustness analysis [36] aiming to determine whether a system is capable to operate when its parameters, initial conditions and/or experimental conditions vary within their expected ranges; determination of modularity of biochemical networks [26] identifying subsets of states whose concentrations depend only on a subset of parameters and identification of species for drug target selection $[31,33]$ by setting the critical steps in the network that could drive disease development.

\section{RESULTS}

In this study, a benchmark problem regarding a biochemical pathway with three enzymatic steps presented by [37] has been considered to illustrate the sensitivity analysis methods described above.

\subsection{Problem statement: three-step biochemical pathway}

This case study, considered as a challenging benchmark problem for parameter estimation by several authors ([38],[39],[40]) involves a biochemical pathway with three enzymatic steps, including the enzymes and mRNAs explicitly. Figure 2 contains a diagram illustrating the network of reactions and kinetic effects (feedback loops). The mathematical formulation of the dynamic model consists

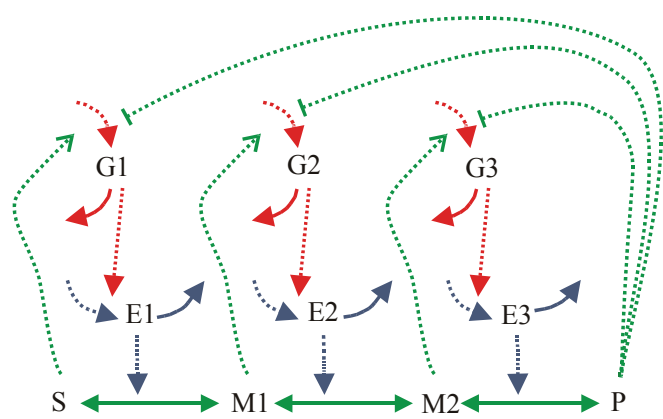

Figure 2. Biochemical pathway scheme. Solid arrows indicate reversible mass transfer reactions, dashed arrows indicate activation, and dashed curves with blunt ends indicate inhibition.

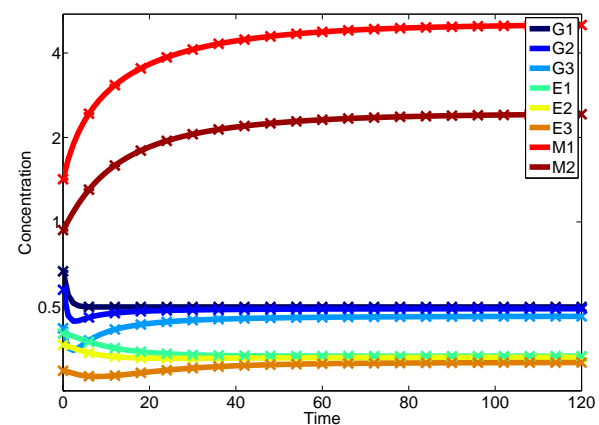

Figure 3. Concentration of the 8 species involved in the model using the nominal values for the parameters. 
of 8 nonlinear ODEs that describe the variation of the metabolite concentration over time:

$$
\begin{aligned}
& \frac{d E_{1}}{d t}=\frac{V_{4} G_{1}}{K_{4}+G_{1}}-k_{4} E_{1} \\
& \frac{d E_{2}}{d t}=\frac{V_{5} G_{2}}{K_{5}+G_{2}}-k_{5} E_{2} \\
& \frac{d E_{3}}{d t}=\frac{V_{6} G_{3}}{K_{6}+G_{3}}-k_{6} E_{3} \\
& \frac{d G_{1}}{d t}=\frac{V_{1}}{1+\left(\frac{P}{K i_{1}}\right)^{n i_{1}}+\left(\frac{K a_{1}}{S}\right)^{n a_{1}}}-k_{1} G_{1} \\
& \frac{d G_{2}}{d t}=\frac{V_{2}}{1+\left(\frac{P}{K i_{2}}\right)^{n i_{2}}+\left(\frac{K a_{2}}{M_{1}}\right)^{n a_{2}}}-k_{2} G_{2} \\
& \frac{d G_{3}}{d t}=\frac{V_{3}}{1+\left(\frac{P}{K i_{3}}\right)^{n i_{3}}+\left(\frac{K a_{3}}{M_{2}}\right)^{n a_{3}}}-k_{3} G_{3} \\
& \frac{d M_{1}}{d t}=\frac{k c a t_{1} E_{1}\left(\frac{1}{K m_{1}}\right)\left(S-M_{1}\right)}{1+\frac{S}{K m_{1}}+\frac{M_{1}}{K m_{2}}}-\frac{k c a t_{2} E_{2}\left(\frac{1}{K m_{3}}\right)\left(M_{1}-M_{2}\right)}{1+\frac{M_{1}}{K m_{3}}+\frac{M_{2}}{K m_{4}}} \\
& \frac{d M_{2}}{d t}=\frac{k c a t_{2} E_{2}\left(\frac{1}{K m_{3}}\right)\left(M_{1}-M_{2}\right)}{1+\frac{M_{1}}{K m_{3}}+\frac{M_{2}}{K m_{4}}}-\frac{k c a t_{3} E_{3}\left(\frac{1}{K m_{5}}\right)\left(M_{2}-P\right)}{1+\frac{M_{2}}{K m_{5}}+\frac{P}{K m_{6}}}
\end{aligned}
$$

where $E_{1}, E_{2}$ and $E_{3}$ are enzymes; $G_{1}, G_{2}$ and $G_{3}$ are the mRNA species for the enzymes; $M_{1}$ and $M_{2}$ are intermediate metabolites of the pathway; $S$ and $P$ (pathway substrate and product) are concentrations under our control (i.e. control variables) and the rest are the kinetic parameters involved on the pathway. The 36 kinetic parameters are divided in two different classes: hill coefficients, allowed to vary within the range $(0.1,10)$, and all the others, allowed to vary within the range $\left(10^{-2}, 10^{2}\right)$. The substrate $(S=10)$ and the product $(P=1)$ are held constant over the experiment. The initial conditions for the remaining states are $E_{1}=4.000, E_{2}=0.3641$, $E_{3}=0.2946, G_{1}=0.6667, G_{2}=0.5725, G_{3}=0.4176, M_{1}=1.419$ and $M_{2}=0.9346$.

The results of an in-silico experiment using the nominal values for the parameters specified in Table I are represented in Figure 3. All the sensitivity metrics were computed for the 36 parameters with respect to the 8 measured states at the measured time points (from $t=0$ to $t=120$, every 6 minutes).

The computations reported in this work were performed on a PC/INTEL XEON CPU (2.13 GHz) running Windows 7 and using the Matlab-based toolbox SensSB [8]. The necessary files to reproduce the results can be found in the following web site: http://www.iim.csic.es/ gingproc/SensSB.html

\subsection{Local sensitivity analysis}

The absolute $\left(S_{a b s}\right)$ and relative $\left(S_{r e l}\right)$ local sensitivity indices were computed at the nominal point for the parameters (see Table I). Figures 4 and 5 show the result of averaging these sensitivities over time for each of the parameters and the contribution of the sensitivities with respect to each of the state variables is represented in different colors. The analysis performed using absolute sensitivities highlights the importance of only 6 of the parameters and most of the uncertainty is generated by the intermediate metabolites $M_{1}$ and $M_{2}$. As can be seen in Figure 3 the absolute value of these two states is way higher than the rest of the species making the absolute sensitivities to have large values too. In contrast, relative sensitivities buffer this effect and reveal the effect of the uncertainty due to the rest of states. This effect would also be found in a parameter estimation problem where the optimization would be mostly driven by fitting these 6 parameters. Thus, there is an obvious motivation for normalizing the models and/or using cost functions for the parameter estimation that 


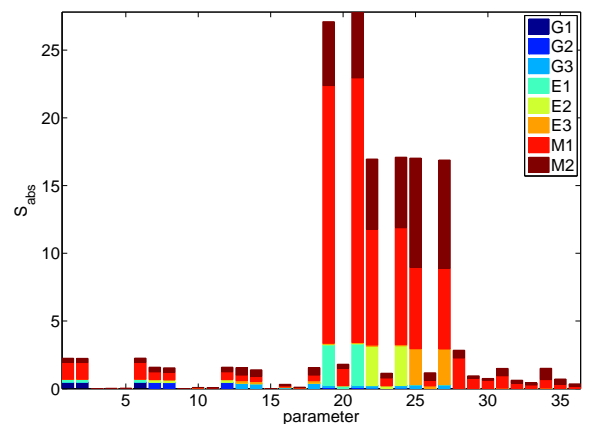

Figure 4. $S_{a b s}$ at the nominal point.

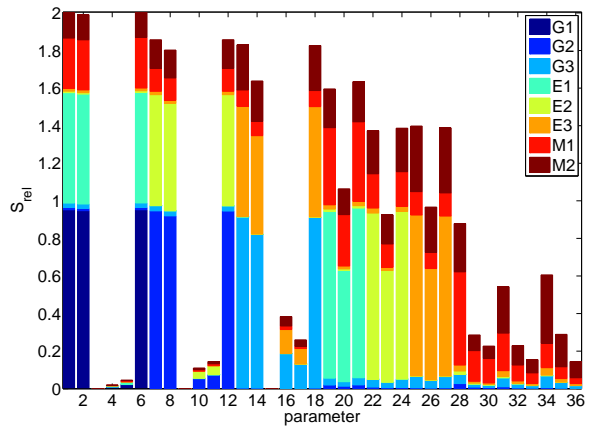

Figure 5. $S_{r e l}$ at the nominal point.

normalize the contribution of the different states as the maximum likelihood estimator of a weighted least squares.

As expected from the model structure they are clusters of parameters influencing each of the state variables, i.e. parameters $p_{1}$ to $p_{6}$ are mainly affecting the dynamics of the $m R N A$ species $G_{1}$ and the enzyme $E_{1}$, parameters $p_{7}$ to $p_{12}$ are mostly influencing $G_{2}$ and $E_{2}$, parameters $p_{13}-p_{18}$ are responsible for $G_{3}$ and $E_{3}$ behavior, $p_{19}-p_{21}$ are involved with the enzyme $E_{1}, p_{22}-p_{24}$ with the enzyme $E_{2}, p_{25}-p_{27}$ with the enzyme $E 3$ and the rest of the parameters are essentially influencing the concentration of the metabolites $M_{1}$ and $M_{2}$ although almost all of the parameters have significant effect on these species what is not surprising since they are intermediate metabolites of the pathway.

The relative sensitivities reveal that for the nominal point, $50 \%$ of the parameters account for less than $15 \%$ of the information while the $20 \%$ most influential account for more than $50 \%$ (see Figure 5). Considering the absolute sensitivities, this effect is even more noticeable (see Figure 4), therefore, in order to efficiently estimate the model parameters most of the efforts should be focussed on the most influential group of parameters whereas the less important can be fixed to their nominal values. These results are also in accordance with the conclusions drawn by Gutenkunst et al. [41] that tested several systems biology models showing that "sloppy" sensitivity spectra (eigenvalues roughly evenly distributed over many decades) are universal in systems biology models. This property helps to explain the difficulty of extracting precise parameter estimates from collective fits and reinforces the need for establishing a parameter ranking.

It can also be seen in Figures 4-5 that parameters $p_{3}, p_{9}$ and $p_{15}$ (i.e. $n i_{1}, n i_{2}$ and $n i_{3}$ ) have negligible sensitivity. This is an obvious result since they are exponents of $\frac{P}{K i_{1}}, \frac{P}{K i_{2}}$ and $\frac{P}{K i_{3}}$ respectively (see Eq. 30-32) and since $P, K i_{1}, K i_{2}$ and $K i_{3}$ are equal to 1 these fractions will not be modified by changes on the exponents. However, this would not be the case if $P$ or $K i_{1}, K i_{2}$ and $K i_{3}$ move from their nominal values what is likely to happen in a real problem where the values of the parameters are usually unknown. This is an artefact of local "one-at-a-time" (OAT) techniques that perturb only one parameter from the nominal point in each simulation missing the effect of perturbing several parameters simultaneously or exploring different regions of the parameter space.

Very valuable information can be drawn from local sensitivities, yet, the most difficult part of the analysis is to identify a good estimator for the parameters since a bad choice of the parameter values will critically influence the parameter ranking. The sensitivity analysis is usually performed before the parameter estimation, therefore, more robust methods are needed in order to ensure that small changes in the parameters values will not significantly change the results of the study.

\subsection{Elementary effect method}

In a first stage, the global sensitivities of the parameters were computed by means of the Morris method using a four level grid $(k=4)$ and 10 trajectories $(N=10)$ as recommended in [1]. For 36 parameters this leads to $N_{F}=370$ function evaluations that for our model can be computed in a few seconds. The low computational cost compared to most of the global sensitivity analysis techniques makes this method very appealing. However, the results obtained were pretty inconsistent 
and different executions of the method provided very different results. Consequently, we increased the number of levels in the grid up to $k=10$ and the number of trajectories to $N=100$ reaching $N_{F}=3700$ that computationally is still affordable (a few minutes in our computer). Figures 6-7 show the results for a uniform and a logarithmic distribution, respectively. The use of a logarithmic distribution allows for a better sampling of the space and more influential parameters were detected. It can also be noticed that even though this is a OAT method, since it is a global technique, the sensitivity for different combinations of parameters can be assessed and $p_{3}, p_{9}$ and $p_{15}$ no longer appear to be negligible as suggested by the local analysis.

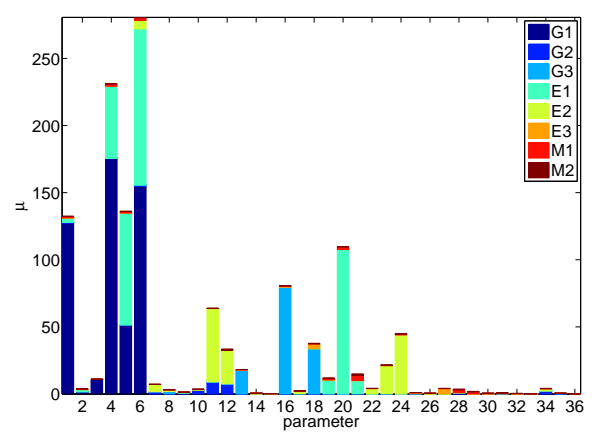

Figure 6. $\bar{\mu}$ with uniform distribution.

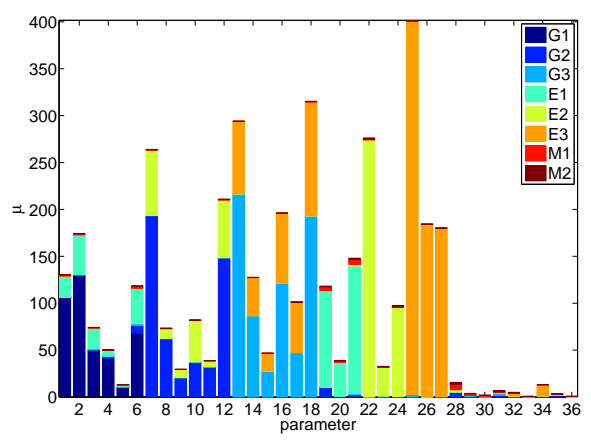

Figure 7. $\bar{\mu}$ with logarithmic distribution.

As will be shown comparing these results with more powerful techniques as variance based ones, the Morris method provides a good compromise between accuracy and efficiency and is very useful as a screening method especially for sensitivity analysis of large or costly models. The overall performance of the measure $\bar{\mu}$ in ranking the factors in order of importance is satisfactory. However, as pointed out by [21], in some instances $\bar{\mu}$ is prone to Type II error, i.e. failing in the identification of factors of considerable influence on the model, being instead more resilient to Type I error, i.e. the erroneous identification of a factor as influential when it is not.

\subsection{Variance based methods}

The Sobol' total sensitivity indices $\left(S I^{T}\right)$ computed from a uniform and a logarithmic distribution using $2^{12}$ LSD points are represented in Figures 8-9. Analogously to the Morris method, the

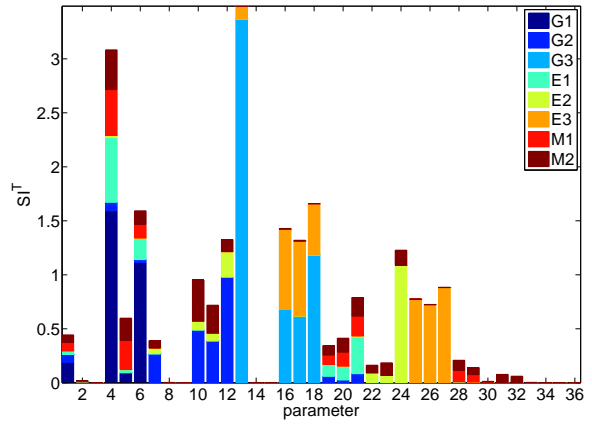

Figure $8 . S I^{T}$ with uniform distribution.

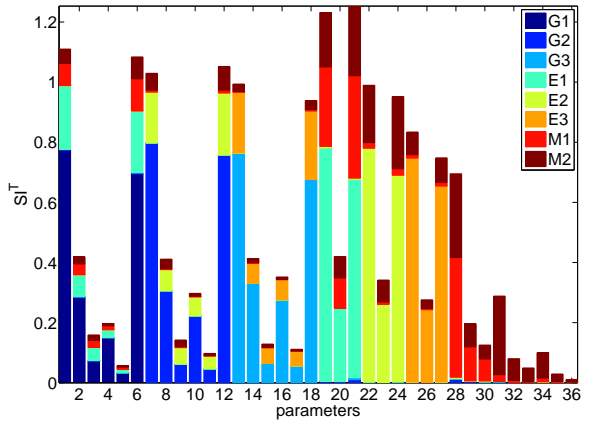

Figure 9. $S I^{T}$ with logarithmic distribution.

logarithmic distribution explores the parameter space in a more effective manner and it detected a larger number of influential parameters. The results from the uniform distribution are pretty different to those obtained by the Morris method but the ones given by the logarithmic distribution are very similar, differing only in the 8 last parameters $\left(p_{28}-p_{36}\right)$ involved mainly with states $M_{1}$ and $M_{2}$. In order to further analyze these differences and to study the influence of averaging the sensitivities 
with respect to the 8 states (Eq. 26) we have computed the partial variances $\left(D_{j}^{T}\right)$ for the logarithmic distribution. The partial derivatives are not normalized by the total sensitivity of each of the outputs, thus, states that have a small contribution to the total uncertainty in absolute terms will not be reflected. Figure 10 shows a very low variance for parameters $p_{28}-p_{36}$ and the contribution of the sensitivity related to states $M_{1}$ and $M_{2}$ is almost negligible. This explains the divergence of the

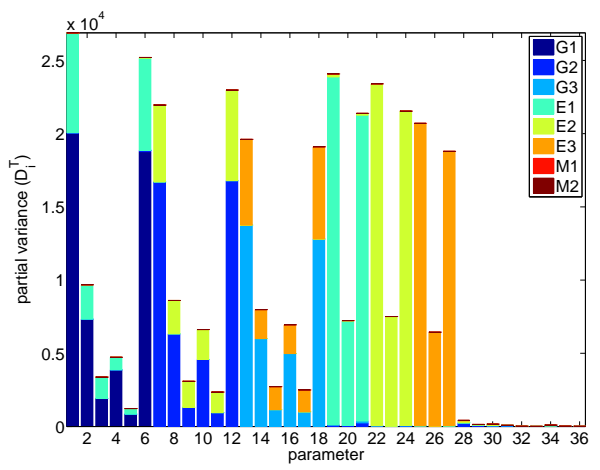

Figure 10. $D^{T}$ with logarithmic distribution.

Sobol' method with the elementary effects based measure $\bar{\mu}$ that, since it is not normalized by the uncertainty of each of the states, also showed very low sensitivity for $p_{28}-p_{36}$.

Sobol' method is a powerful technique for quantifying the relative importance of input factors or groups of factors. However, the main drawback is the computational cost associated that, for computationally intensive models or models with a large number of parameters, can become prohibitive. The convergence properties of this method will be analyzed in more detail in section 3.6.

\subsection{Derivative based global sensitivity measures}

Figures 11-12 represent the values of the derivative based sensitivity measure $M_{r e l}$ based on relative sensitivities and the uncertainty due to each of the state variables, computed using $2^{12}$ LSD points. In this case, the uniform distribution captures better the uncertainty than the Morris and Sobol'

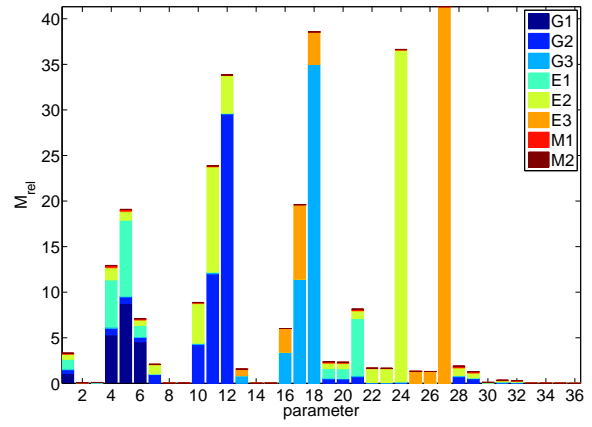

Figure 11. $M_{r e l}$ with uniform distribution.

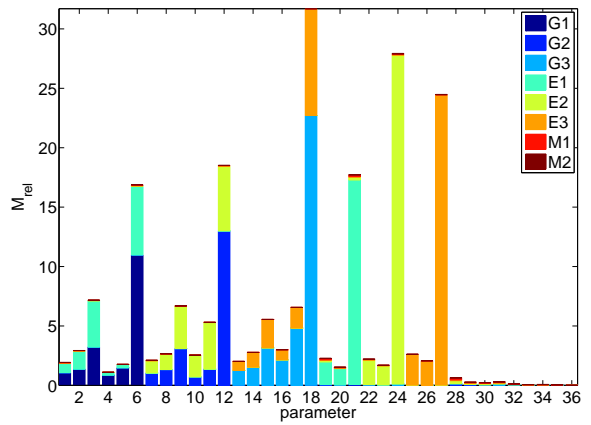

Figure 12. $M_{r e l}$ with logarithmic distribution.

methods using the same distribution function. Nonetheless, the logarithmic distribution identified a larger set of sensitive parameters. The results of the DGSM are quite different to the Sobol $S^{T}$ and the Morris $\bar{\mu}$ although the three methods are able to identify the most important factors. The main reason for that is that DGSM relies on partial derivatives while Sobol and Morris methods are based on incremental ratios. The parameters with the highest $M_{r e l}$ are $p_{6}, p_{12}, p_{18}, p_{21}, p_{24}$ and $p_{27}$ that in the model could be seen as the eigenvalues of the system. When considering a large domain for 
the parameters, DGSM give valuable information about the structure of model without taking into account the uncertainty of each of the parameter (i.e. parameters playing the same role in the model equations but with different distribution would not be distinguished).

The relative sensitivities for parameters $p_{28}-p_{36}$ and the contribution of the states $M_{1}$ and $M_{2}$ that were significant for the nominal point are negligible in the overall parameter space in agreement with the results provided by $\bar{\mu}$ and $D^{T}$.

This structural information can be very useful, however, computational researchers are often interested in the sensitivity in a plausible region of the parameter space. In this case, and when some experimental information is available, the use Bayesian DGSM is advisable. Figure 13 shows the distribution for parameters $p_{1}$ and $p_{2}$. In this case we have a convex problem with enough information to define the distribution, thus, the measure $M_{r e l, B}$ tends to the local sensitivity values in the optimal set of parameters (see Figure 14). This method is advantageous since it does not

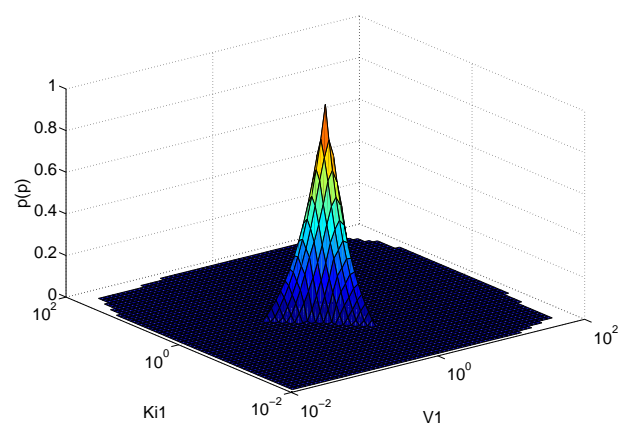

Figure 13. Distribution of parameters $p_{1}$ and $p_{2}$.

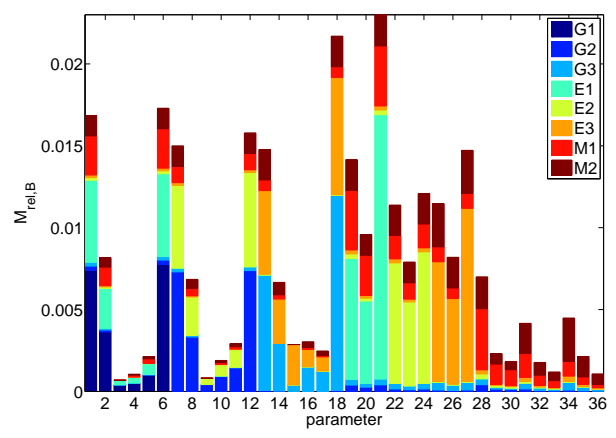

Figure 14. $M_{r e l, B}$ with logarithmic distribution.

require a previous estimation of the parameters and it also allows the consideration of functions that either due to the lack of information or structural properties have multiple minima in the area of interest and therefore they do not have a convex distribution function.

\subsection{Convergence of the numerical methods}

In order to analyze the convergence properties of Sobol' and DGSM methods, the results obtained from Monte Carlo and Quasi Monte Carlo (Sobol' LDS) based integration are compared here. Considering the sensitivity obtained with the maximum number of sampling points $\left(2^{n}\right)$ as the true value, the error of the method is computed as Eq. 35:

$$
\operatorname{error} S I\left(2^{i}\right)=\sqrt{\left(\frac{S I\left(2^{i}\right)-S I\left(2^{n}\right)}{S I\left(2^{n}\right)}\right)^{2}} \text { for } i=1: n
$$

The convergence considering the results from $2^{13}$ LSD points as the true value is represented in Figures 15-16 for Sobol' and DGSM methods respectively, plotting the evolution of the error with respect to the number of sampling points. Note that each evaluation of a sampling point for Sobol' indices implies the computation of the model $N_{p}+2$ times (in this case 38 times) while the evaluation of a sampling point for DGSM only requires the computation of the partial derivatives with respect to each parameter.

From Figures 15-16 it follows that QMC integration provides very fast convergence rate with -1.08 for $S I^{T}$ and -0.58 for $M_{r e l}$, while for MC integration convergence is much slower with -0.58 for $S I^{T}$ and -0.26 for $M_{r e l}$. Apart from DGSM using MC integration, the rest of the methods achieved a convergence error lower than $10 \%$. Although this error could be improved by increasing the number of sampling points, we consider this value acceptable for our purposes. This convergence analysis is of great importance for determining the sufficient number of sampling points required to properly evaluate the sensitivities since it is problem dependent and no a priori value can be suggested. 


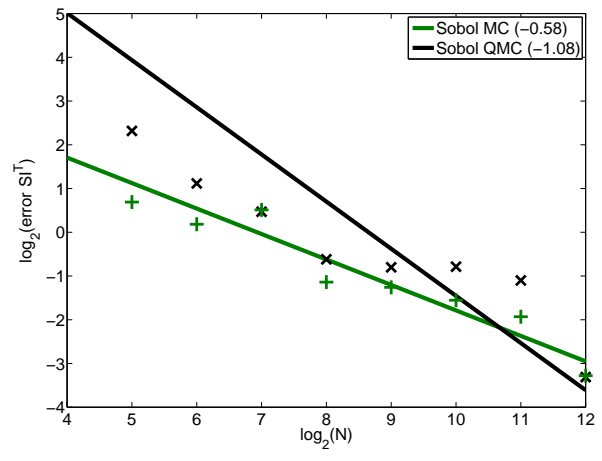

Figure 15. Convergence of $S I_{i j}^{T}$.

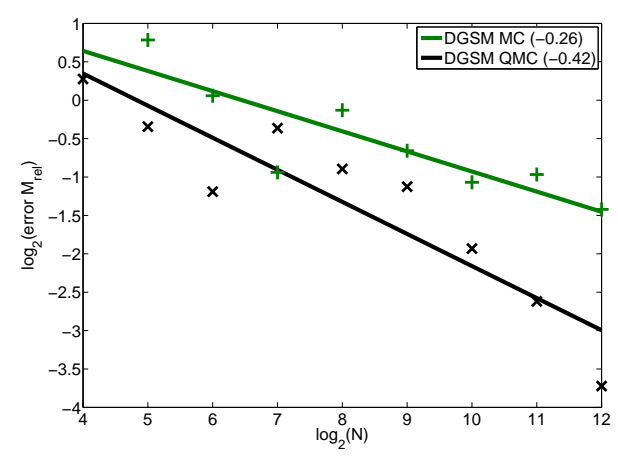

Figure 16. Convergence of $M_{\text {rel }}$.

\section{CONCLUSIONS}

Global sensitivity analysis is an emerging tool for assisting in the development of sound dynamic models of biological systems. An overview of some of the available methodologies is provided here and the main reasons for their analogies and differences are analyzed.

The Morris method provides a good compromise between accuracy and efficiency and it is particularly useful for computationally expensive models. The choice of $k=4$ and $N=10$ recommended in the literature has been found insufficient for the model considered in this work and a thinner grid together with a larger number of trajectories is suggested in order to highly improve the accuracy maintaining efficiency.

The importance of analyzing the partial derivatives $D^{T}$ together with the Sobol' indices is highlighted in this work. The need for summarizing the information about the sensitivity with respect to different states can sometimes lead to indices of difficult interpretation and some of the information can be lost in the normalization process. The values of $D^{T}$ can provide information about the uncertainty whereas $S I$ and $S I^{T}$ are only focused on the sensitivity (how this uncertainty is distributed among the different input factors).

Derivative based Global Sensitivity Measures(DGSM) provide information about the model structure and are less dependent on the distribution of the parameters. Moreover, DGSM are ideal to study how the model is affected by small changes in the input factors while the Morris and Sobol' methods are preferred when we are interested on analyzing the results of larger alterations.

The critical influence of the parameters distribution on the results of a sensitivity analysis is stated and illustrated with a relevant benchmark model. The choice of a logarithmic distribution for exploring large parameter spaces without prior information about the plausible regions for the optimal values has been proven very effective for the three global methods considered (Morris method, Sobol' indices and DGSM). Moreover, the QMC based integration method showed higher convergence rate than the MC for computing the Sobol' indices and derivative based global sensitivity measures.

The novel methodology presented here, Bayesian DGSM, provides a good estimation of the sensitivity of the parameters taking maximum advantage of the a priori available information in form of experimental data.

\section{APPENDIX A}

\section{ACKNOWLEDGEMENT}

MRF and FJD acknowledge the financial support provided by the Institute for Collaborative Biotechnologies 
Table I. Nominal values and bounds for the 36 kinetic parameters.

\begin{tabular}{|c|c|c|c|c|c|}
\hline Parameter & $p \#$ & Nom. val & Parameter & $p \#$ & Nom. val \\
\hline$V_{1}$ & $p_{1}$ & 1 & $V_{4}$ & $p_{19}$ & 0.1 \\
\hline$K i_{1}$ & $p_{2}$ & 1 & $K_{4}$ & $p_{20}$ & 1 \\
\hline$n i_{1}$ & $p_{3}$ & 2 & $k_{4}$ & $p_{21}$ & 0.1 \\
\hline$K a_{1}$ & $p_{4}$ & 1 & $V_{5}$ & $p_{22}$ & 0.1 \\
\hline$n a_{1}$ & $p_{5}$ & 2 & $K_{5}$ & $p_{23}$ & 1 \\
\hline$k_{1}$ & $p_{6}$ & 1 & $k_{5}$ & $p_{24}$ & 0.1 \\
\hline$V_{2}$ & $p_{7}$ & 1 & $V_{6}$ & $p_{25}$ & 0.1 \\
\hline$K i_{2}$ & $p_{8}$ & 1 & $K_{6}$ & $p_{26}$ & 1 \\
\hline$n i_{2}$ & $p_{9}$ & 2 & $k_{6}$ & $p_{27}$ & 0.1 \\
\hline$K a_{2}$ & $p_{10}$ & 1 & kcat $_{1}$ & $p_{28}$ & 1 \\
\hline$n a_{2}$ & $p_{11}$ & 2 & $K m_{1}$ & $p_{29}$ & 1 \\
\hline$k_{2}$ & $p_{12}$ & 1 & $K m_{2}$ & $p_{30}$ & 1 \\
\hline$V_{3}$ & $p_{13}$ & 1 & kcat $_{2}$ & $p_{31}$ & 1 \\
\hline$K i_{3}$ & $p_{14}$ & 1 & $K m_{3}$ & $p_{32}$ & 1 \\
\hline$n i_{3}$ & $p_{15}$ & 2 & $K m_{4}$ & $p_{33}$ & 1 \\
\hline$K a_{3}$ & $p_{16}$ & 1 & kcat $_{3}$ & $p_{34}$ & 1 \\
\hline$n a_{3}$ & $p_{17}$ & 2 & $K m_{5}$ & $p_{35}$ & 1 \\
\hline$k_{3}$ & $p_{18}$ & 1 & $K m_{6}$ & $p_{36}$ & 1 \\
\hline
\end{tabular}

through grants No. W911NF-07-1-0279 and W911NF-09-D-0001 from the U.S. Army Research Office. Author JRB acknowledges support by the Spanish government, MICINN project "MultiSysBio" (ref. DPI2008-06880-C03-02), and by CSIC intramural project "BioREDES" (ref. PIE-201170E018). The authors thank the reviewers for their valuable comments and suggestions.

\section{REFERENCES}

1. Saltelli A, Ratto M, Andres T, Campolongo F, Cariboni J, Gatelli D, Saisana M, Tarantola S. Global sensitivity analysis: the primer. John Wiley \& Sons Ltd., 2008.

2. Saltelli A, Tarantola S, Campolongo F, Ratto M. Sensitivity analysis in practice: a guide to assessing scientific models. John Wiley \& Sons Ltd., 2004.

3. van Riel NAW. Dynamic modelling and analysis of biochemical networks: mechanism-based models and modelbased experiments. Briefings in Bioinformatics 2006; 7(4):364-374.

4. Schmidt H, Jirstrand M. Systems biology toolbox for MATLAB: a computational platform for research in systems biology. Bioinformatics 2006; 22(4):514-515.

5. Hoops S, Sahle S, Gauges R, Lee C, Pahle J, Simus N, Singhal M, Xu L, Mendes P, Kummer U. COPASI-a COmplex PAthway SImulator. Bioinformatics 2006; 22(24):3067-3074.

6. Zi Z, Zheng Y, Rundell AE, Klipp E. SBML-SAT: a systems biology markup language (SBML) based sensitivity analysis tool. BMC Bioinformatics 2008; 9:342.

7. Maiwald T, Timmer J. Dynamical modeling and multi-experiment fitting with PottersWheel. Bioinformatics 2008; 24(18):2037-2043.

8. Rodriguez-Fernandez M, Banga JR. SensSB: a software toolbox for the development and sensitivity analysis of systems biology models. Bioinformatics 2010; 26(13):1675-1676.

9. Zheng Y, Rundell AE. Comparative study of parameter sensitivity analyses of the TCR-activated erk-MAPK signalling pathway. IEE Proceedings Systems Biology 2006; 153(4):201-11.

10. Bagheri N, Stelling J, Doyle III FJ. Quantitative performance metrics for robustness in circadian rhythms. Bioinformatics 2007; 23(3):358-364.

11. Kiparissides A, Kucherenko SS, Mantalaris A, Pistikopoulos EN. Global sensitivity analysis challenges in biological systems modeling. Industrial \& Engineering Chemistry Research 2009; 48(15):7168-7180.

12. Helton JC. Uncertainty and sensitivity analysis techniques for use in performance assessment for radioactive waste disposal. Reliability Engineering \& System Safety 1993; 42(2-3):327 - 367.

13. Egea JA, Rodriguez-Fernandez M, Banga JR, Marti R. Scatter Search for chemical and bio-process optimization. Journal of Global Optimization 2007; 37:481-503.

14. Morris MD. Factorial sampling plans for preliminary computational experiments. Technometrics 1991; 33(2):161174. 
15. Sobol' IM. Global sensitivity indices for nonlinear mathematical models and their monte carlo estimates. Mathematics and Computers in Simulation 2001; 55:271-280.

16. Kucherenko S, Rodriguez-Fernandez M, Pantelides C, Shah N. Monte Carlo evaluation of derivative based global sensitivity measures. Reliability Engineering \& System Safety 2009; 94:1135-1148.

17. Versyck K. Dynamic input design for optimal estimation of kinetic parameters in bioprocess models. PhD Thesis, Katholieke Universiteit Leuven 2000.

18. Saltelli A, Chan K, Scott EM. Sensitivity Analysis. John Wiley \& Sons Ltd., 2000.

19. Maly T, Petzold LR. Numerical methods and software for sensitivity analysis of sifferential-algebraic systems. Applied Numerical Mathematics 1996; 20:57-79.

20. Leis JR, Kramer MA. ODESSA - an ordinary differential-equation solver with explicit simultaneous sensitivity analysis. ACM Transactions on Mathematical Software 1988; 14(1):61-67.

21. Campolongo F, Cariboni J, Saltelli A. An effective screening design for sensitivity analysis of large models. Environmental Modelling \& Software 2007; 22(10):1509-1518.

22. Saltelli A, Tarantola S, Chan KPS. A quantitative model-independent method for global sensitivity analysis of model output. Technometrics 1999; 41(1):39-56.

23. Saltelli A. Making best use of model evaluations to compute sensitivity indices. Computer Physics Communications 2002; 145(2):280-29.

24. Sobol M, Kucherenko S. Derivative based global sensitivity measures and their link with global sensitivity indices. Mathematics and Computers in Simulation 2009; 79:3009-3017.

25. Cho KH, Shin SY, Kolch W, Wolkenhauer O. Experimental design in systems biology, based on parameter sensitivity analysis using a Monte Carlo method: a case study for the TNF $\alpha$-mediated NF- $\kappa \mathrm{B}$ signal transduction pathway. Simulation 2003; 79:726-739.

26. Bentele M, Lavrik I, Ulrich M, Stober S, Heermann DW, Kalthoff H, Krammer PH, Eils R. Mathematical modeling reveals threshold mechanism in CD95-induced apoptosis. Journal of Cell Biology 2004; 166(6):839-851.

27. Aldrich J. R. A. Fisher and the making of maximum likelihood 1912-1922. Statistical Science 1997; 12(3):162-176.

28. Walter E, Pronzato L. Identification of Parametric Models from Experimental Data. Springer, 1997.

29. Sobol I. On quasi monte carlo integrations. Mathematics and Computers in Simulation 1998; 47:103-112.

30. Zhang HX, Dempsey Jr WP, J G. Probabilistic sensitivity analysis of biochemical reaction systems. Journal of Chemical Physics 2009; 131(9):094 101.

31. Feng XJ, Hooshangi S, Chen D, Li G, Weiss R, Rabitz H. Optimizing genetic circuits by global sensitivity analysis. Biophysical Journal 2004; 87(4):2195 - 2202.

32. Kontoravdi C, Asprey SP, Pistikopoulos EN, Mantalaris A. Application of global sensitivity analysis to determine goals for design of experiments: an example study on antibody-producing cell cultures. Biotechnology Progress $2005 ; 21(4): 1128-1135$.

33. Sahle S, Mendes P, Hoops S, Kummer U. A new strategy for assessing sensitivities in biochemical models. Philosophical Transactions of the Royal Society A 2008; 366:3619-3631.

34. Yue H, Brown M, He F, Jia J, Kell DB. Sensitivity analysis and robust experimental design of a signal transduction pathway system. International Journal of Chemical Kinetics 2008; 40(11):730-741.

35. Yuraszeck TM, Neveu P, Rodriguez-Fernandez M, Robinson AE, Kosik KS, Doyle III FJ. Vulnerabilities in the tau network and the role of ultrasensitive points in tau pathophysiology. PLoS Computational Biology 2010; In press.

36. Gunawardena J. Elements of Computational Systems Biology, chap. Models in systems biology: the parameter problem and the meanings of robustness. John Wiley and Sons Ltd., 2009; 19-47.

37. Mendes P. Foundations of Systems Biology., chap. Modeling Large Biological Systems from Funcational Genomic Data: Parameter Estimation. MA: MIT Press, Cambridge, 2001; 163-186.

38. Moles C, Mendes P, Banga J. Parameter estimation in biochemical pathways: a comparison of global optimization methods. Genome Research 2003; 13:2467-2474.

39. Rodriguez-Fernandez M, Mendes P, Banga J. A hybrid approach for efficient and robust parameter estimation in biochemical pathways. BioSystems 2006; 83:248-265.

40. Liu PK, Wang FS. Hybrid differential evolution with geometric mean mutation in parameter estimation of bioreaction systems with large parameter search space. Computers \& Chemical Engineering 2009; 33(11):1851 -1860 .

41. Gutenkunst RN, Waterfall JJ, Casey FP, Brown KS, Myers CR, Sethna JP. Universally sloppy parameter sensitivities in systems biology models. PLOS Computational Biology 2007; 3(10):1871-1878. 